\title{
UTILIZAÇÃO DE COMPUTAÇÃO MÓVEL PARA QUALIFICAÇÃO DE ROTINAS DE OPERAÇÃO E MANUTENÇÃO DE REDES DE DISTRIBUIÇÃO
}

\author{
Carlos O. Pretto* \\ Mauro A. da Rosa*
}

Flávio A. Becon Lemos*

Tiago T. dos Santos*

${ }^{*}$ Grupo de Sistemas de Energia Elétrica - GSEE

Faculdade de Engenharia - PUCRS

Av. Ipiranga 6681, Prédio 30, Partenon

CEP 90619-900 - Porto Alegre RS

\section{RESUMO}

O objetivo deste artigo é discutir a utilização de um conjunto de tecnologias de computação móvel (Mobile Computing) para auxílio nas rotinas de planejamento da operação e manutenção de sistemas de distribuição de energia elétrica. Apresenta-se um resumo das principais tecnologias, discutindo-se definições, serviços e aplicações em potencial na área de sistemas de energia. Para ilustrar o artigo é apresentado um caso de utilização prática da tecnologia de Personal Digital Assistant - PDA, para coleta e validação de dados de eventos não programados em sistemas de distribuição, um sistema para auxílio de rotinas de inspeção e manutenção em redes de distribuição e um sistema de comunicação de dados via telefonia celular para uma pequena central hidrelétrica.

PALAVRAS-CHAVE: computação móvel, palm-top, planejamento da operação, planejamento da manutenção.
Artigo submetido em 30/12/2004

1a. Revisão em 05/01/2006

2a. Revisão em 21/10/2006

Aceito sob recomendação do Editor Associado Prof. Carlos A. Castro

\section{ABSTRACT}

This paper presents an overview on use of mobile computing to support routines of operation and maintenance planning of electrical distribution systems. A review of main technologies is presented, together with a discussion about definitions, services and applications of each one. Some potential utilisation in power system is described. The paper is illustrated with a practical implementation of Personal Digital Assistant - PDA technology to collecting and validation of outage events in electrical distribution systems, another system to aid inspections and maintenance routines using PDA and a communication system to a small hydropower plant using mobile phone network.

KEYWORDS: Mobile computing, personal digital assistant PDA, distribution planning, distribution operation.

\section{INTRODUÇÃO}

A reformulação do setor elétrico brasileiro, iniciada nos anos 90, culminou com a privatização de várias empresas, com destaque para as empresas de distribuição de energia elétrica, e inseriu novos conceitos de competição, produtividade e desempenho na distribuição de energia. A nova estrutura do setor elétrico impõe às distribuidoras regras de forneci- 
mento com alto grau de segurança, qualidade e continuidade, as quais são fiscalizadas pela Agência Nacional de Energia Elétrica - ANEEL. O ambiente atual requer das empresas a busca pela eficiência econômica (Billinton et al, 2002) e pela melhoria do desempenho dos índices de continuidade e tensão. Toda essa nova estrutura passa pela revisão dos processos internos das empresas, objetivando suprimir práticas ultrapassadas, muitas das quais baseadas nas experiências dos técnicos e na confecção manual de relatórios, por processos suportados por metodologias automatizadas que agilizam e fornecem confiabilidade às informações. A necessidade de rever e aprimorar esses processos se justifica, não só pelo aumento da eficiência das empresas, como pela fiscalização e pelas penalidades impostas pela ANEEL às empresas pelo não cumprimento de requisitos de qualidade do serviço prestado. Além disso, o código de defesa do consumidor também impõe à empresa a necessidade de prestar um serviço dentro de padrões aceitáveis de qualidade.

Dentro deste contexto, a utilização de computação e comunicação móvel contribui para aumentar e qualificar a eficiência dos processos envolvidos na aquisição, validação e análise dos processos de inspeção de redes, tanto os associados a manutenções preventivas quanto aos associados a manutenções corretivas (Pretto et al., 2003b) (Silva et al., 2003). Isso implica na padronização e qualificação do processo de coleta de informações, tanto de forma manual, com auxílio de computadores móveis, como de forma automatizada, utilizando sistemas remotos de aquisição de dados. Esse tipo de processo diminui a ocorrência de erros devido às falhas humanas no preenchimento de questionários, identificação de causas de eventos, erros em inspeções de campo, em rotinas de manutenção e aumenta a disponibilidade de informações importantes para o funcionamento do sistema através da aquisição remota.

Tecnologias de computação e comunicação móvel estão em contínuo avanço em termos de disponibilidade, funcionalidade e custos, tornando-se atraentes para os planos de automatização das empresas. Essas tecnologias permitem uma grande variedade de aplicações, sendo a escolha de uma ou outra, ponderada pelas características do problema. Dentre essas tecnologias pode-se citar o Personal Digital Assistant - PDA, conhecido como Palm-Top, e a comunicação de dados via rede de telefonia celular.

Este artigo apresenta uma revisão sobre as principais tecnologias de computação móvel e telefonia celular que podem suportar aplicações na área de sistemas de energia, bem como apresenta exemplos práticos de aplicações dessas tecnologias. Na seção 2 é apresentada a motivação da utilização de computação móvel no atual cenário do setor elétrico. A seção 3 apresenta alguns conceitos fundamentais de telefonia celular, bem como os principais tipos de computadores de mão atualmente utilizados. Para ilustrar este artigo, as seções 4 e 5 apresentam duas aplicações baseadas em PDAs e a seção 6 uma aplicação baseada em comunicação de dados via telefonia celular (Santos et al., 2004). As principais conclusões do artigo são apresentadas na seção 7 .

\section{MOTIVAÇÃO}

No atual cenário do setor elétrico é importante que o fornecimento de energia seja efetuado com um alto grau de confiabilidade e qualidade, tal que satisfaça os anseios do consumidor e atenda aos padrões de desempenho de continuidade e tensão impostos pelo órgão regulador. Estas condições exigem um melhoramento contínuo nas rotinas de inspeção de redes, uma vez que ações de planejamento da manutenção e rotinas de operação sofrem uma grande influência da qualidade das informações obtidas nestes processos. Desta forma, é importante procurar apoio em modernas tecnologias para aprimorar esses processos, de forma a proporcionar mais agilidade, facilidade e qualidade na coleta de dados, segurança no armazenamento dos dados e velocidade na transmissão de informações. Uma tecnologia disponível para esse aprimoramento é a da computação móvel, que envolve desde computadores de mão até telefones celulares. Sendo assim, são apresentados três exemplos de aplicações que utilizam computação móvel e telefonia celular para o aprimoramento de processos de operação e manutenção de sistemas elétricos. O primeiro refere-se ao uso de um computador de mão para coleta de dados de desligamentos não programados. Dentre os inúmeros processos que ocorrem paralelamente em uma distribuidora de energia, pode-se considerar o tratamento de desligamentos não programados como de fundamental importância para garantir um bom desempenho do sistema. Considera-se aqui, o tratamento de eventos não programados, como tudo que envolve o processamento das informações sobre este tipo de desligamento, desde a coleta das informações no local do evento, identificação da sua causa, armazenamento das informações, até o tratamento dessas informações para futuras inferências. Neste artigo é dado ênfase ao processo de coleta de dados com a utilização de um computador de mão, auxiliando o eletricista na coleta de informações sobre os eventos não programados, uma vez que esses dados indicam onde determinado tipo de evento possui maior nível de ocorrência, orientando as ações de manutenção e balizando o planejamento dos investimentos em expansão. O segundo exemplo consiste em um sistema de auxílio nas rotinas de inspeção e manutenção através de um aplicativo gráfico de representação da rede de distribuição em um computador de mão. O terceiro corresponde a um sistema de aquisição de dados de pequenas centrais hidrelétricas utilizando a rede celular de telefonia. 


\section{PRINCIPAIS TECNOLOGIAS DE COMU- NICAÇÃO E MOBILIDADE}

Empresas de energia elétrica viam a telefonia fixa e os enlaces dedicados de rádio-frequiência como únicas soluções viáveis para a comunicação de dados entre os centros de operações e os equipamentos remotos. Devido aos altos custos envolvidos na implantação e manutenção de tais sistemas, se tornava inviável a automatização de um grande número de pontos dentro de um sistema de distribuição de energia elétrica. Porém, nos últimos dez anos, a rede pública de telefonia celular e a comunicação via satélite apresentaram avanços significativos em relação a sua disponibilidade, viabilidade e abrangência para novas aplicações. Algumas das principais tecnologias desenvolvidas para comunicação e mobilidade atualmente disponíveis no mercado são apresentadas abaixo.

\subsection{AMPS (Advance Mobile Phone Sys- tem)}

O sistema AMPS é um sistema de telefonia celular analógico, denominado de primeira geração. É o mais antigo e difundido sistema de telefonia celular, desenvolvido pelo Bell Labs na década de 80. Fornece serviço de voz modulado em uma portadora FM, num canal de rádio freqüência. Esse sistema permite a transmissão de dados na forma CSD (Circuit Switched Data), mas com um desempenho geralmente descrito como marginal, sendo muito menor do que em canais discados convencionais, ficando limitado a taxas de $10 \mathrm{kbps}$. Conforme a presença de obstáculos a taxa de transmissão pode cair para $1 \mathrm{kbps}$. Outro inconveniente desse tipo de sistema é a facilidade com que o mesmo possa ser fraudado. Porém, esse sistema apresenta uma vantagem no que diz respeito à área de cobertura, possibilitando acesso a pontos distantes de centros urbanos (Siemens AG, 2003).

\subsection{IS-136}

Tecnologia de segunda geração pioneira na transmissão de voz e dados digitalmente. Utiliza duas técnicas de acesso aos domínios tempo e frequencia chamadas de TDMA (Time Division Multiple Access) e FDMA (Frequency Division Multiple Access). Através dessas técnicas, a banda disponível é dividida em canais de $30 \mathrm{kHz}$, onde o acesso ao domínio temporal é dividido em quadros de $40 \mathrm{~ms}$. Cada quadro de $40 \mathrm{~ms}$ é dividido em 6 slots de $6,67 \mathrm{~ms}$. Como cada chamada telefônica utiliza dois slots, torna-se possível que até 3 conversações sejam estabelecidas em um único canal com banda de $30 \mathrm{kHz}$. O IS-136 possibilitou que novos serviços fossem disponibilizados, tais como transferência de dados utilizando CSD a uma velocidade de 9,6 kbps, identificação do número chamador, conferência, siga-me e chamada em espera.

\section{$3.3 \quad$ IS-95}

Tecnologia digital, classificada como de segunda geração, criada no início dos anos 90 . Utiliza uma técnica de acesso múltiplo por divisão de código (CDMA - Code Division Multiple Access), sendo esse código a identificação de cada celular no sistema. Permite transmissão de dados utilizando CSD a uma taxa de 14,4 kbps, SMS e 1XRTT (1xRadio Transmission Technology) a uma taxa média de 57,6 kbps (Siemens AG, 2003).

\subsection{GSM (Global System for Mobile Com- munication)}

O sistema GSM é um padrão de telefonia celular de segunda geração criado com o propósito de dar origem a um sistema de telecomunicações europeu operativo na freqüência de 900 $\mathrm{MHz}$, encontrando-se hoje expandido além das fronteiras européias. As bandas do GSM são divididas em canais de RF, onde cada canal consiste de um par de freqüências (Transmissão e Recepção) com $200 \mathrm{KHz}$ de banda cada. Existem, portanto, 124 canais de RF no GSM 900 e 373 canais no DCS 1800. Esses canais receberam uma numeração conhecida como ARFCN (Absolute Radio Frequency Channel Number). As frequiências portadoras dos canais de RF são moduladas em 0,3 GMSK por um sinal digital com taxa de $270,833 \mathrm{kbit} / \mathrm{s}$. Este sinal digital de 270,833 kbit/s é dividido no domínio do tempo em 8 intervalos (slots) de tempo possibilitando o múltiplo acesso por divisão no tempo (TDMA) das Estações Móveis. O padrão GSM permite a transferência de dados utilizando as tecnologias CSD, SMS, GPRS e EDGE (Siemens AG, 2003).

\subsection{Tecnologias de Comunicação de Da- dos}

Cada tipo de rede celular tem seus padrões de transferência de dados. Estas tecnologias podem ser utilizadas nas mais diversas áreas de sistemas de potência, tais como: monitoração de demanda em pontos estratégicos, telecomando de religadores e banco de capacitores, operação remota da geração, supervisão de subestações, controle da frota de veículos da empresa e até mesmo coleta de registros para tarifação de consumidores. Abaixo são apresentadas as principais tecnologias de transferência de dados disponíveis atualmente no Brasil (Siemens AG, 2003).

\subsubsection{CSD (Circuit Switched Data)}

É um sistema de transmissão de dados onde a transferência é feita através de um canal de voz comum (comutação por circuito). O inconveniente deste tipo de sistema é a tarifação, pois como o sistema reserva um canal de voz para tráfego de 
dados, o mesmo é tarifado por pulsos telefônicos pela operadora. As velocidades de transferência de dados ficam em torno de 9,6 kbps para as redes do tipo AMPS e TDMA e 14,4 kbps para as redes GSM e CDMA (Siemens AG, 2003). Um exemplo de aplicação desse sistema de transferência de dados é descrito na seção 6 (Santos et. al., 2004).

\subsubsection{SMS (Short Message Service)}

É um serviço de envio de menssagens instantâneas que começou a ser disponibilizado nos sistemas digitais de segunda geração. Permite envio e recebimento de mensagens com até 160 caracteres. Se o telefone de destino estiver fora da área de cobertura ou desligado, as mensagens são guardadas por um centro de mensagens que evita que as mesmas sejam perdidas (Siemens AG, 2003). A utilização deste tipo de serviço na área de sistemas de potência é restrita, pois as operadoras não garantem o tempo de entrega das mensagens, podendo gerar atrasos indesejáveis dependendo da aplicação.

\subsubsection{GPRS (General Packet Radio Server)}

Tecnologia de transmissão de dados baseada na rede GSM, que faz comutação por pacotes. Uma empresa pode utilizar o serviço de transferência de dados independentemente da utilização do serviço de voz, pagando uma taxa proporcional ao volume de dados necessário para a aplicação (Siemens AG, 2003). Como em sistemas de telemetria, a taxa de dados trafegados é baixa, se comparada a outras aplicações, tal tecnologia se torna atraente. Uma aplicação deste tipo de tecnologia em sistemas de potência é descrito na seção 6 (Santos et. al., 2004).

\subsubsection{EDGE (Enhanced Data Rates for GSM Evolu- tion)}

Padrão desenvolvido para aumentar a taxa de transferência de dados pela rede GSM. Desta maneira é possível oferecer taxas de transferência de dados a taxas de até 384 kpbs. Sua forma de tarifação é semelhante à do GPRS, onde o usuário paga por Mbyte trafegado durante o mês (Siemens AG, 2003).

\subsubsection{XRTT (1xRadio Transmission Technology)}

É uma tecnologia que permite a transferência de dados em alta velocidade no padrão de rede CDMA. É um equivalente do GPRS, permitindo transferência de dados entre 30 a 70 kbps, sendo tarifado da mesma forma (Siemens AG, 2003).
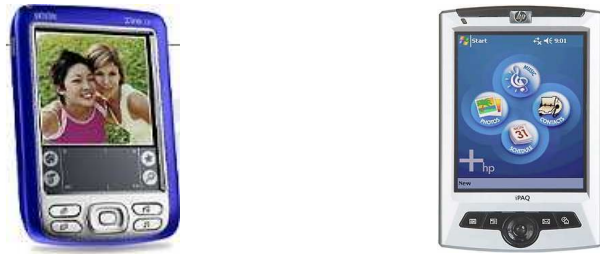

Figura 1: Exemplos de PDAs
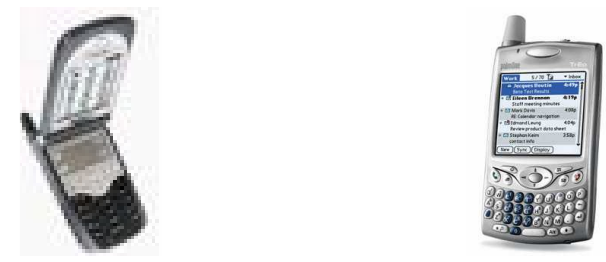

Figura 2: Exemplos de SmartPhone e WDA

\subsection{Computadores Móveis}

\subsubsection{PDA (Personal Digital Assistant)}

PDAs são dispositivos portáteis com tela monocromática ou colorida e sistema de entrada de dados por tela sensível ao toque (touch screen). Exemplos de PDA são mostrados na figura 1. Normalmente os sistemas operacionais presentes já incluem aplicativos como calendário, agenda, bloco de notas entre outros. Existem ferramentas comerciais e livres para desenvolvimento de aplicativos para estas plataformas. A interface física com outros equipamentos pode ser realizada através de comunicação serial, bluethooth e por alguns slots do tipo PCMCIA e compact flash. Existem comercialmente, interfaces com estes dispositivos para a utilização de transferência de dados via GPRS e também utilizando serviços da rede CDMA. Os dois principais sistemas operacionais para PDAs são o Palm OS da Palm Inc e o Pocket PC da Microsoft ${ }^{\circledR}$ Inc (CIGRE, 2002).

\subsubsection{Smartphones}

Os Smartphones são telefones celulares com função de PDA, como os mostrados na figura 2. Atualmente a maior desvantagem é o tamanho da tela, a qual, geralmente, é menor que a de um PDA. Os Smartphones com o mesmo tamanho de tela de um PDA são chamados de WDA (Wireless Digital Assistants). A convergência entre os smarthphones e os PDAs é considerada natural (CIGRE, 2002).

\subsubsection{Handheld PC}

O handheld PC, figura 3, é similar ao PDA no sentido que eles são terminais com tela colorida e sistema de entrada de 


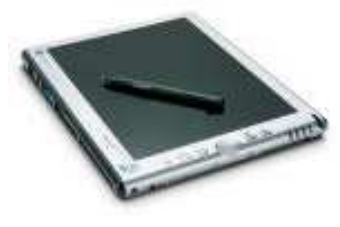

Figura 3: Handheld PC

dados por tela sensível ao toque (touch screen), porém com um tamanho de tela bem maior, geralmente com a resolução de 800 x 600 pixels ou mais. Os handhelds utilizam os mesmos sistemas operacionais que um PC de mesa, e costumam ser chamados também de Tablet PC (CIGRE, 2002).

\subsubsection{Terminais Dedicados}

Terminais com interface gráfica geralmente baseados em tela de cristal líquido (LCD - Liquid Crystal Display) monocromático alfanumérico e sem a capacidade de programação pelo usuário. Um exemplo disso são os sistemas via satélite para coleta de dados (CIGRE, 2002).

\subsubsection{Telefones Celulares}

Uma das tecnologias de computação móvel mais difundida atualmente são os telefones celulares, os quais apresentam um baixo custo de aquisição, ampla cobertura de serviços e uma grande potencialidade de utilização como dispositivo de aquisição de dados. Outra grande vantagem do telefone celular é permitir a sua utilização para comunicação de voz e dados. Geralmente possuem telas coloridas, porém com tamanho reduzido, o que muitas vezes limita o tipo de aplicação que pode ser suportada pelo dispositivo. Alguns possuem funções extras como câmera fotográfica, o que permite o desenvolvimento de aplicativos com capacidade de armazenamento fotográfico. Alguns dispositivos utilizam o sistema operacional Symbian OS ${ }^{\circledR}$. Outros utilizam uma máquina virtual Java para executar aplicativos desenvolvidos por terceiros (CIGRE, 2002).

\section{SISTEMA PARA AQUISIÇÃO DE DADOS DE EVENTOS NÃO PROGRAMADOS}

Atualmente os operadores e eletricistas são os analistas dos defeitos que ocorrem nas redes elétricas. Muitas vezes não há possibilidade de identificação direta da causa que originou a parada no fornecimento de energia. A correta identificação da causa de um evento está diretamente relacionada à quantidade e à qualidade da informação no local do evento, bem como da identificação correta das atividades e dos fatos que poderiam indicar a causa de uma falta de energia não programada. Para auxiliar o eletricista nesta atividade, foi desen-
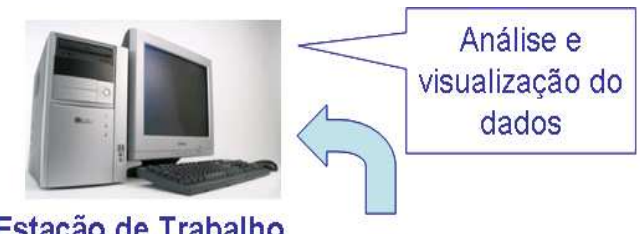

Estação de Trabalho

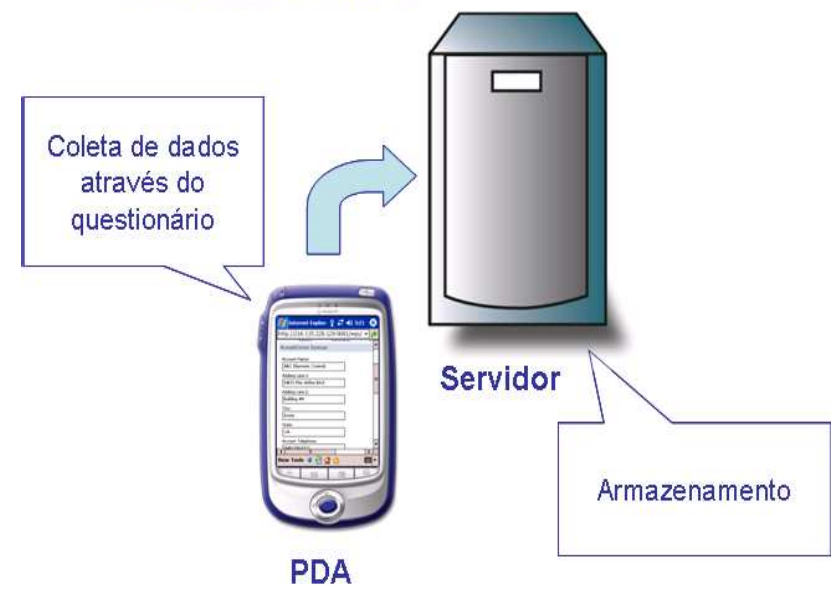

Figura 4: Arquitetura do sistema de coleta de dados de eventos não programados

volvido um sistema de coleta de dados com a utilização de tecnologia móvel do tipo PDA, cuja arquitetura simplificada é apresentada na figura 4. Nesse sistema, o eletricista deve escolher a resposta mais apropriada para traduzir as condições do elemento do sistema de distribuição que falhou e as variáveis relativas às condições climáticas na hora da ocorrência do evento. Esse sistema de coleta de dados pode ser considerado o primeiro módulo de um sistema de tratamento das informações de falta de energia. O segundo módulo consiste em um aplicativo que irá analisar as informações colhidas em campo pelo computador de mão e, através de um algoritmo computacional, identificar as possíveis causas do evento (Pretto at al, 2003a).

\subsection{Sistema de Coleta}

O objetivo da detecção da causa (Billinton et al., 2002) de paralisação do fornecimento de energia é a de suprir as áreas da empresa que gerencia a rede elétrica, com informações sobre o desempenho dos elementos que compõem as mesmas, identificando como posicionar o investimento necessário para redução de problemas e conseqüente ampliação de benefícios (Billinton et al., 1991). Nesse caso, é necessário identificar os elementos básicos de um sistema de distribuição para melhor medir o desempenho desses elementos. Sendo assim, pode-se citar alguns objetivos básicos de interesse nos elementos, sendo eles: 
- Identificar quais áreas possuem piores desempenhos das redes;

- Identificar qualidade dos equipamentos instalados no sistema de distribuição;

- Identificar possibilidade de mau gerenciamento dos ativos por parte dos técnicos que atuam na rede;

- Identificar o desempenho de algum tipo de equipamento que seja necessário em alguma região;

- Identificar problemas de redes elétricas mal construídas.

\subsubsection{Elementos de Interrupção}

As falhas que ocorrem em um sistema de distribuição acontecem diretamente em algum dos elementos que compõem a estrutura física da rede elétrica. Baseado nisso, criou-se o conceito de "elemento de interrupção", representando todas as estruturas que podem apresentar defeitos e, consequentemente, levar à interrupção do fornecimento de energia elétrica (Rosa et al., 2004). Os elementos de interrupção podem ser identificados como:

- Postes;

- Cruzetas;

- Isoladores;

- Elementos de Sustentação;

- Condutores;

- Equipamentos.

Utilizando esse conceito de elemento de interrupção, foi elaborado um questionário que visa identificar o motivo da paralisação do fornecimento de energia elétrica. O questionário foi implementado sob forma de um aplicativo para computador de mão, onde o eletricista indica o elemento de interrupção responsável pela parada de fornecimento e as condições onde a rede de distribuição esta inserida.

O fluxo da informação e a sequiência lógica do questionário podem ser vistos na figura 5 , onde o fluxo começa com a chegada de uma Ordem de Serviço - OS.

\subsubsection{Implementação do Aplicativo de Coleta}

O questionário foi implementado sob forma de um aplicativo desenvolvido em uma estrutura orientada a objetos, onde os dados recolhidos são armazenados em um arquivo local para

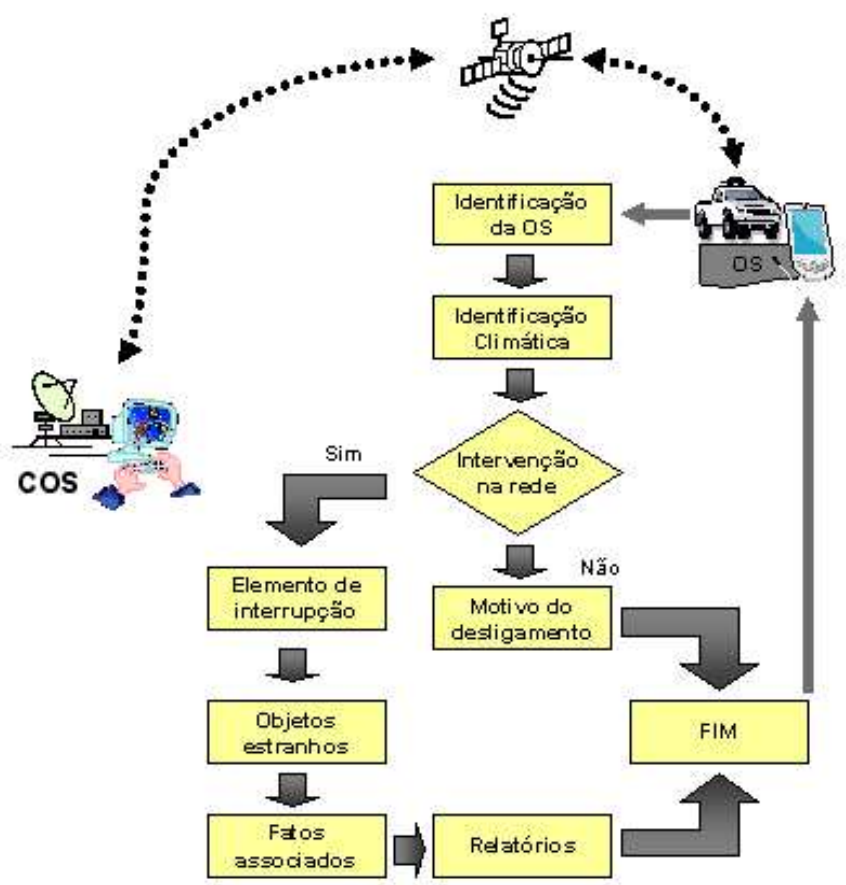

Figura 5: Seqüência lógica do questionário

posteriormente ser enviado a um banco de dados. É importante salientar que o principal objetivo do eletricista é restaurar o sistema de distribuição, restabelecendo o fornecimento de energia o mais breve possível. Sendo assim, o questionário deve ser respondido após a intervenção na rede, com o eletricista possuindo claro domínio sobre o trabalho realizado. O questionário deve possuir um conteúdo claro, intuitivo e de fácil navegação. O questionário foi implementado de três formas diferentes. A primeira versão foi implementada com o uso da linguagem Java através da API MIDP, para celulares e computadores portáteis. A segunda implementação foi realizada com a utilização da linguagem Embedded Visual Basic ${ }^{\circledR}$, para a plataforma Pocket $P C{ }^{\circledR}$, e por fim, a terceira implementação valeu-se dos recursos da linguagem de programação $\mathrm{C}++$ para realizar o desenvolvimento do aplicativo compatível com o sistema operacional Palm OS ${ }^{\mathbb{R}}$. A seguir é feita uma breve descrição dos detalhes de cada desenvolvimento e, posteriormente, uma comparação entre os mesmos.

\subsubsection{Java}

A linguagem Java é uma linguagem multiplataforma onde os aplicativos são executados por uma máquina virtual. $\mathrm{O}$ compilador Java gera um bytecode que é interpretado por uma máquina virtual específica para cada sistema operacional. Existe uma máquina virtual Java para a maioria dos computadores portáteis e telefones móveis atuais, e isto pos- 


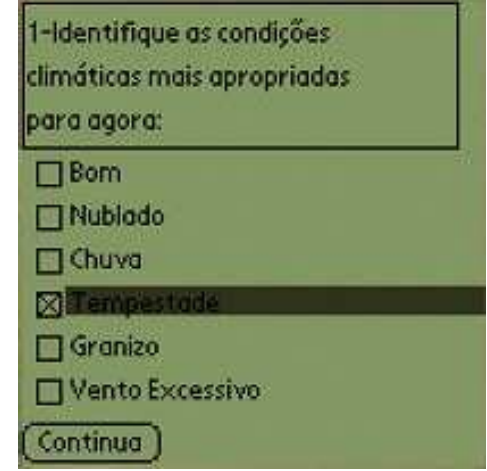

Figura 6: Tela clima

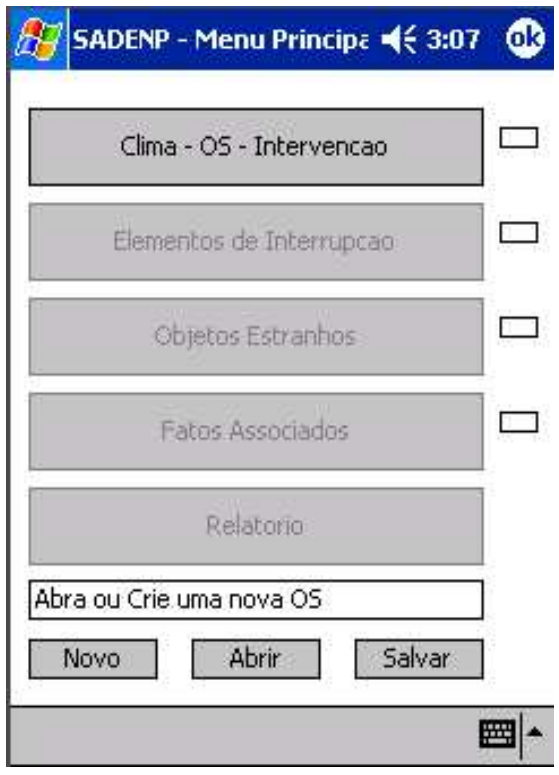

Figura 7: Aplicativo para Pocket $\mathrm{PC}{ }^{\circledR}$

sibilitou o desenvolvimento do aplicativo utilizando essa linguagem. Toda a interface homem-máquina (IHM) do aplicativo foi "desenhada" utilizando a classe Canvas com os métodos de desenho apropriados. O usuário podia entrar com os dados com o uso da caneta touch screen ou através das teclas de acesso. Na figura 6 pode ser vista uma tela do sistema desenvolvido em Java para Palm OS ${ }^{\circledR}$ (Pretto et al.,2003a).

\subsubsection{Embedded Visual Basic}

A empresa Microsoft disponibiliza gratuitamente a ferramenta Embedded Visual Basic para desenvolvimento a aplicativos voltados aos sistemas operacionais Pocket PC e Windows CE. Essa ferramenta permite o desenvolvimento rápido de aplicativos, com várias ferramentas para construção de IHM. Na figura 7 é mostrada uma tela do aplicativo desenvolvido para o sistema operacional Pocket PC.

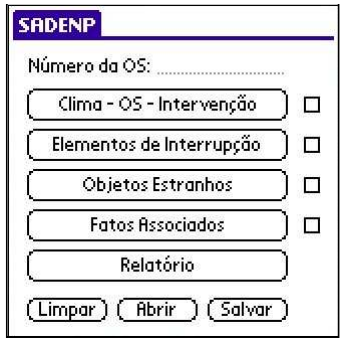

Figura 8: Tela inicial do aplicativo

\begin{tabular}{|c|c|}
\hline \multicolumn{2}{|l|}{ SADENP - Clima } \\
\hline \multicolumn{2}{|l|}{ Clima Atual: } \\
\hline Condiç̃o: & Vento: \\
\hline - Condiçăo & - Vento \\
\hline \multicolumn{2}{|l|}{ Clima Evento: } \\
\hline Condiģôno: & Vento: \\
\hline - Condição & - Vento \\
\hline \multicolumn{2}{|c|}{$\square$ Interwenção na Rede? } \\
\hline Cancelar & Confirmar \\
\hline
\end{tabular}

Figura 9: Tela de identificação climática

\subsubsection{C++}

Utilizou-se a linguagem C++ (Meiler et al.,2001) com o uso da ferramenta de desenvolvimento CodeWarriror ${ }^{\circledR}$ para desenvolver uma versão definitiva do aplicativo de coleta, com todas as funcionalidades necessárias para o sistema operacional Palm OS ${ }^{\circledR}$. O uso dessa ferramenta se mostrou o mais vantajoso das utilizadas, pois apresentou uma boa velocidade de desenvolvimento com recursos ilimitados de programação. O desempenho obtido foi muito satisfatório, proporcionando uma interação rápida e eficiente do aplicativo com o usuário. Nas figuras 8 e 9 são mostradas telas do aplicativo desenvolvido em $\mathrm{C}++$.

Nas figuras 10 e 11 é apresentado um exemplo de um atendimento utilizando o aplicativo desenvolvido para PDA.

\subsubsection{Vantagens e Desvantagens}

Uma das fases críticas no desenvolvimento de aplicativos para dispositivos de computação móvel é a escolha da linguagem de programação e da plataforma de desenvolvimento. Essa escolha deve levar em conta algumas características fundamentais, tais como velocidade de desenvolvimento, portabilidade e documentação. A tabela 1 apresenta uma comparação entre três diferentes linguagens de programação utilizadas no desenvolvimento dos aplicativos para computadores móveis.

A linguagem Java apresenta uma velocidade de desenvolvimento elevada, pois é totalmente orientada a objetos, promo- 

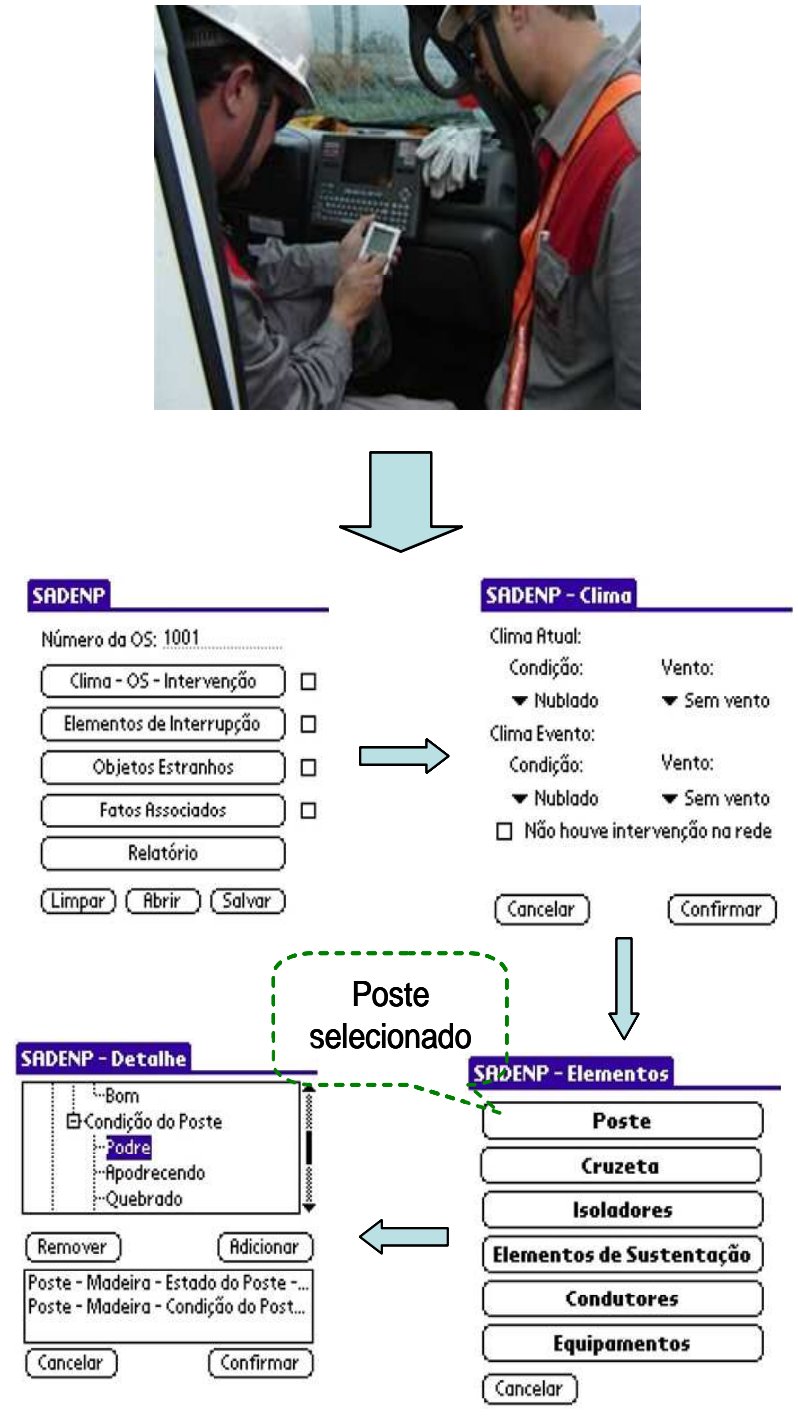

Figura 10: Primeira parte do aplicativo.

vendo a reutilização de classes para a realização das mais diversas funções. A ferramenta que utiliza C++ gera códigos nativos para a maioria dos processadores da linha de PDA da Palm ${ }^{\circledR}$, sendo muito eficiente para aplicações que requerem desempenho avançado. A linguagem Embedded Visual Basic ${ }^{\circledR}$ é gratuita, pode ser usada tanto para desenvolvimento para Pocket $P C^{\circledR}$ quanto para Windows $C E^{\circledR}$, é de fácil entendimento, porém sem portabilidade para sistemas que utilizem Palm OS ${ }^{\circledR}$.

\subsection{Transferência de Dados}

O processo de comunicação entre PDA - PC é uma peça fundamental no sistema portátil, uma vez que realiza as transações entre ambas as plataformas. O mecanismo responsável

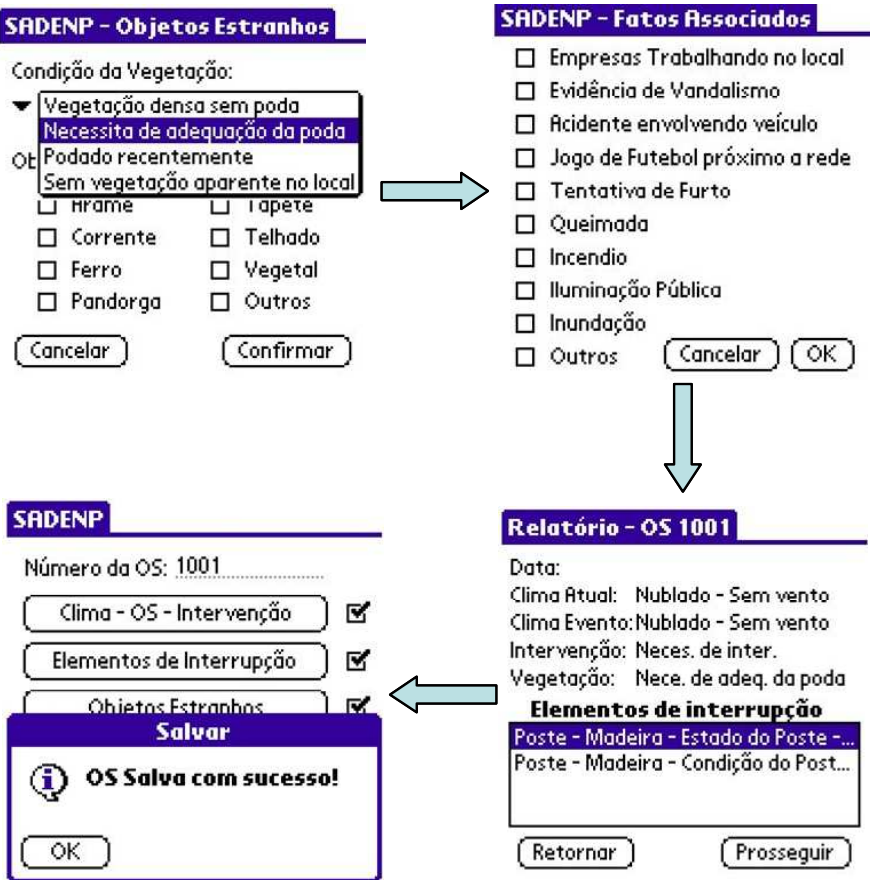

Figura 11: Segunda parte do aplicativo.

\begin{tabular}{|c|c|c|c|c|}
\hline Linguagem & Custo & $\begin{array}{c}\text { Velocidade de } \\
\text { desenvolvimento }\end{array}$ & Portabilidade & Documentação \\
\hline Java & Zero & Rápida & Total & Muito Boa \\
\hline VB & Zero & Rápida & Nula & Média \\
\hline $\begin{array}{c}\text { C++ } \\
\text { (CodeWarrior) }\end{array}$ & $\begin{array}{c}+/- \\
\text { U } \$ 200^{*}\end{array}$ & Média & Parcial & Muito Boa \\
\hline
\end{tabular}

*Fonte: www.metrowerks.com

Tabela 1: Comparativo entre linguagens de programação para computadores móveis

por esse processo deve ser robusto, para evitar inconsistências. Contudo, não deve ser um aplicativo complexo.

O sistema operacional Palm OS ${ }^{\circledR}$ possui um programa de comunicação padrão chamado HotSync ${ }^{\circledR}$, desenvolvido pela própria Palm ${ }^{\circledR}$. A estrutura de sincronismo deste software é bastante simples. O dispositivo Palm ${ }^{\circledR}$ solicita a sincroniza - troca de informações entre PDA e PC - e o aplicativo gerente de comunicação, instalado no PC, responde a este chamado, executando as tarefas programadas em bibliotecas chamadas de Conduits. Cada aplicação no dispositivo Palm ${ }^{\circledR}$ tem um respectivo Conduit que gerencia a lógica de transferência de dados tal como as suas consistência, como apresentado na Figura 12.

Para a criação de Conduits, existe um pacote de desenvolvimento disponibilizado pela Palm OS ${ }^{\circledR}$ chamado Conduit Development Kit - CDK. Este pacote consiste em uma sé- 


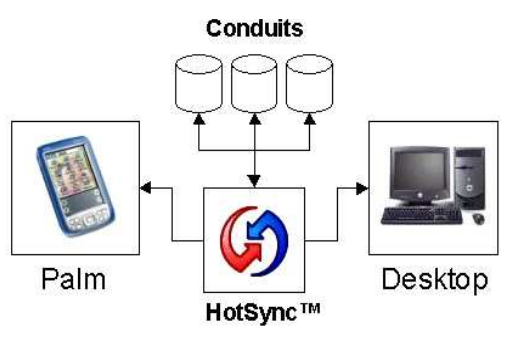

Figura 12: Conduits

rie de bibliotecas e interfaces de tratamento de requisições e de comunicação para interação com o software HotSync ${ }^{\circledR}$. Uma solução de comunicação utilizando o CDK foi implementada e testada, porém foi descartada devido a necessidade da instalação do framework .NET nas máquinas que realizariam a sincronização.

Sendo assim, buscou-se outra solução para a implementação da comunicação. Esta solução foi baseada na utilização em conjunto de bibliotecas de Conduits para a linguagem Delphi ${ }^{\circledR}$ e o uso de uma linguagem de colagem para gerenciamento da lógica de transferência de dados. A liguagem de colagem escolhida foi a Python, devido a sua facilidade de imlpementação.

Utilizando a flexibilidade da linguagem juntamente com um pacote de bibliotecas chamado Python For Delphi $($, foi possível integrar as duas linguagens utilizando a primeira como linguagem de colagem e a segunda como gerenciadora do Conduit para desenvolver a terceira versão do sistema de comunicação. Esta última versão trouxe flexibilidade para o sistema, sem perda de desempenho ou estabilidade.

O processo de comunicação alterou-se na parte relativa a interação entre HotSync $囚$ e Conduit. Antes era estabelecido um pareamento entre aplicativos e Conduits, pois cada programa exigia uma lógica diferente de trabalho de dados. Agora, existe uma duplicidade entre programas e arquivos de script em Python ${ }^{\circledR}$, onde cada arquivo (em formato texto) descreve a lógica relativa a um dado programa, e apenas um Conduit que gerencia as execuções necessárias, como apresentado esquematicamente na Figura 13.

\subsection{Armazenamento}

Os dados coletados em campo são armazenados em um banco de dados Oracle ${ }^{\circledR}$ ligado a outras bases de dados da empresa, formando um sistema de informação que pode fornecer as mais diversas consultas de interesse para aprimoramento das políticas de planejamento e expansão (Pretto et. al., 2003). Essa estrutura de armazenamento dos dados permite que o operador relacione os dados dos eventos com as mais diversas variáveis de interesse. O operador pode, por

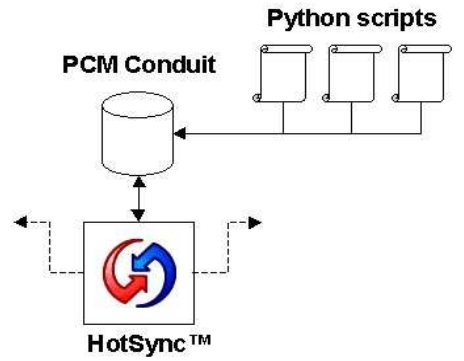

Figura 13: Delphi - Python

exemplo, relacionar dados de utilização de material com os atendimentos dos eventos não programados, pode relacionar a freqüência de ações de poda de árvores com dados dos desligamentos ocorridos por influência de vegetais, a concentração de eventos em determinadas áreas, entre outros.

\subsection{Análise dos Dados}

O aplicativo de análise tem por objetivo proporcionar um meio de visualização dos dados sobre desligamentos, tornando-se uma ferramenta muito útil para o operador. A visualização de dados sob a forma gráfica é um recurso amplamente utilizado para identificação de padrões, os quais normalmente não são vistos quando os dados estão dispostos de maneira tabular. O processo de análise dos dados inicia com a escolha de um período para a pesquisa no banco de dados do histórico de falhas. Considerando que o número de interrupções não programadas em um ano é da ordem de milhares, é importante limitar a pesquisa para um determinado período de interesse. Após a escolha do período de análise, o aplicativo fornece a visualização gráfica das ocorrências sobre o diagrama unifilar do alimentador bem como o cruzamento de dados para estudos. O aplicativo traz alguns benefícios diretos através de seu uso, sendo os mais significativos:

Manutenção - Possibilidade de verificar graficamente as áreas da rede que apresentam um maior tipo de defeito, isso pode ser feito através de uma pesquisa filtrada pelo item Elemento de Interrupção.

Áreas de Poda - Verificar os locais que ocorrem mais desligamentos associados ao item vegetal. Esta tarefa pode ser realizada através de uma pesquisa filtrada pelo item Vegetal. Este estudo pode descobrir que zonas precisam de uma rotina de poda mais freqüente.

Estudo Elétrico - O eletricista pode reproduzir o estado elétrico da rede no momento de qualquer interrupção presente no histórico de interrupções, verificando as condições de corrente e tensão no local da falha.

Estado Elétrico - O aplicativo pode apresentar que parte da 


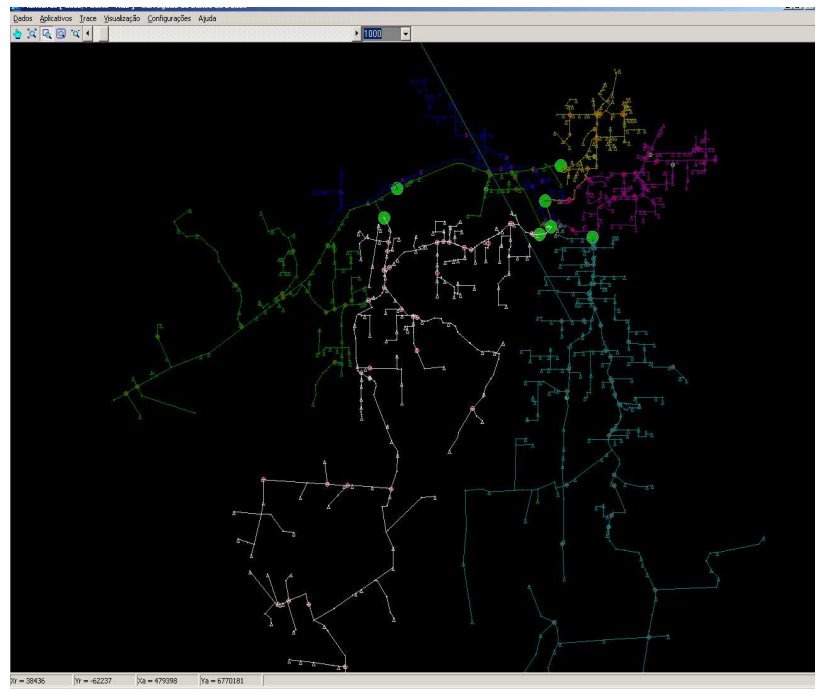

Figura 14: Saída gráfica do aplicativo de análise.

rede ficou sem abastecimento depois de um desligamento e por quanto tempo.

Inspeção - O aplicativo pode servir como apoio a políticas de inspeção de rede, baseado no histórico de falhas, no tipo de falha, e na frequiência que cada tipo de equipamento apresenta defeito.

A Figura 14 mostra um exemplo de saída gráfica do aplicativo de análise, onde é possível observar círculos desenhados sobre o diagrama unifilar da rede de distribuição. Esses círculos representam interrupções não programadas de energia que aconteceram naquele local, em um período e em um equipamento determinado.

\subsection{Implantação e Aceitação do Sistema}

O sistema foi bem aceito pela empresa e obteve uma excelente repercussão, uma vez que contribuiu diretamente para o aumento da qualidade da informação. A empresa agora conta com uma ferramenta que pode orientar, de maneira pontual e focada, os investimentos realizados na rede. As funcionalidades de visualização gráfica agradam o operador, que consegue visualizar com clareza as variáveis e os padrões decorrentes do histórico de desligamento, podendo guiar ações para aumentar a confiabilidade do sistema, com clareza e precisão.

\section{INSPEÇÃO DE REDE COM PDA}

A manutenção de um sistema elétrico é fator essencial na produtividade e competitividade das empresas de energia elétrica, uma vez que a qualidade e a continuidade são direta-

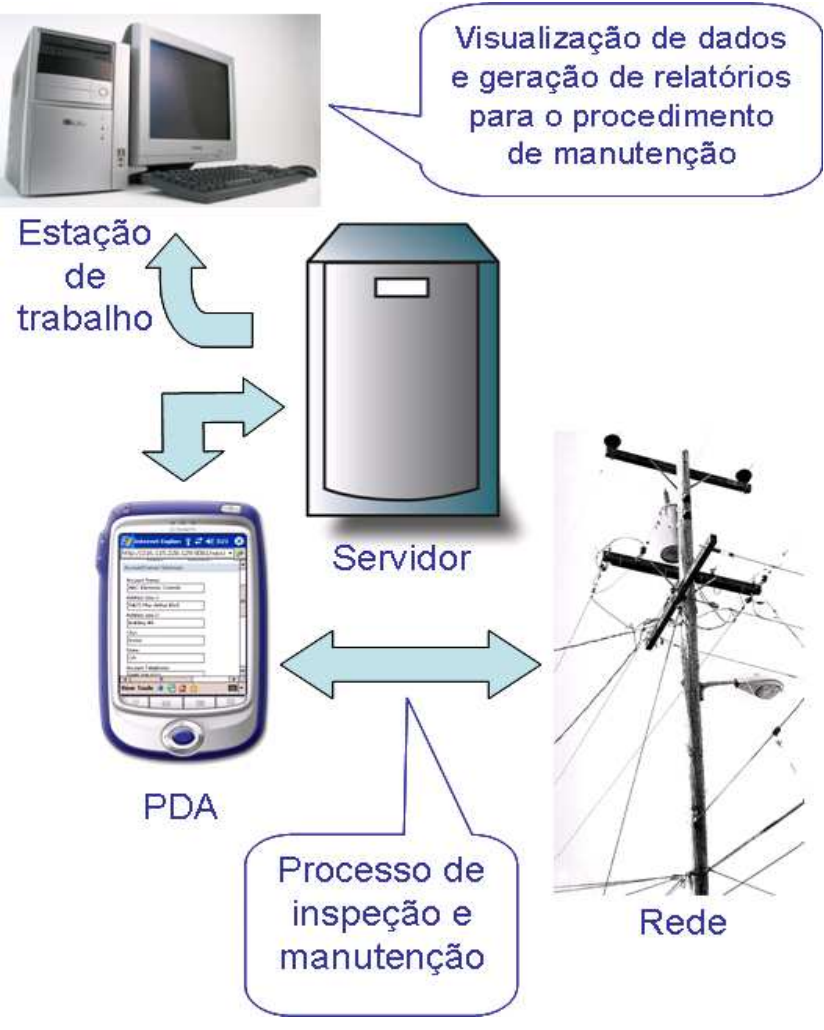

Figura 15: Arquitetura lógica do sistema de inspeção de redes

mente influenciadas pelo desempenho dos equipamentos da rede (Endrenyi et al., 2001). A tarefa de inspeção de redes é uma área promissora para a utilização de computadores móveis. Dois tipos de aplicações de sistemas de inspeção utilizando computação móvel são relatados em (Silva et al., 2003) e (Monteiro et al.,2003). Na seção 5.1 é apresentado um sistema desenvolvido e implantado pelos autores em uma empresa de distribuição de energia elétrica.

\subsection{Sistema}

Na figura 15 pode ser visto um esquemático que demonstra a arquitetura proposta. O eletricista utiliza o PDA para indicar através de uma tree view os equipamentos que necessitam de uma ação de manutenção, além de indicar quais ações são necessárias e o seu grau de prioridade. $\mathrm{O}$ aplicativo proporciona uma visão georeferenciada da rede elétrica na tela do dispositivo e o usuário pode identificar o local onde é necessário realizar a inspeção. Na figura 16 pode ser vista a representação de um alimentador real na tela de um dispositivo da Palm ${ }^{\circledR}$. Na figura 17 pode ser visto um exemplo da tree view de equipamentos com suas respectivas ações e prioridades. 


\section{CELESC}

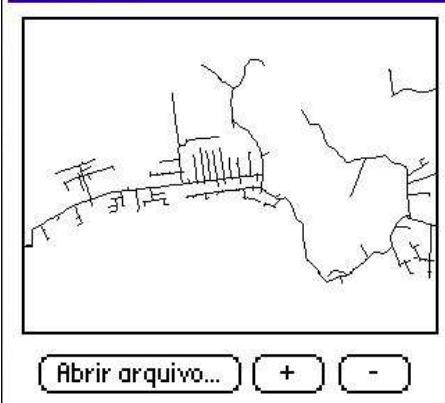

Figura 16: Apresentação do alimentador na tela do PDA

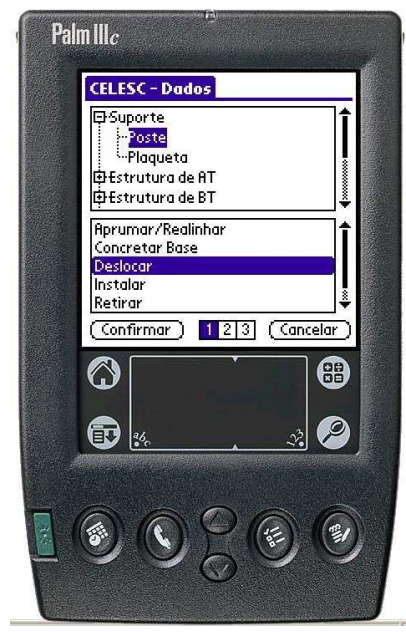

Figura 17: Árvore de componentes

\subsection{Desenvolvimento Computacional}

Para a implementação das rotinas gráficas do aplicativo destinado ao PDA foram criadas duas entidades, sendo elas: nó e ramo. Cada nó possui um identificador (ID) específico e suas respectivas coordenadas $\mathrm{x}$ e y. O ramo é composto por um identificador (ID), um nó inicial e um nó final. Esta estruturação viabiliza a criação de um vínculo entre uma região física do alimentador com coordenadas geográficas e as estruturas nela presentes. Desta forma é possível vincular um ponto geográfico às estruturas presentes neste ponto, possibilitando ao usuário acessar os conjuntos de estruturas através de uma representação gráfica da rede. $\mathrm{O}$ alimentador representado na Figura 16, por exemplo, contém mais de 2000 nós, e aproximadamente a metade desse valor de ramos. Sendo assim a manipulação desse volume de dados pelo dispositivo portátil torna-se penosa. Baseado nisso, todo o desenvolvimento foi realizado em $\mathrm{C}++$, pois foi a linguagem que apresentou o melhor desempenho para a solução proposta.

\section{AQUISIÇÃO DE DADOS DE PCH}

Nesta seção é descrito um sistema para aquisição de dados de pequenas centrais hidrelétricas através da rede de telefonia celular.

\subsection{Introdução}

Durante muitos anos no Brasil, pequenos aproveitamentos energéticos de base hidráulica, as chamadas Pequenas Centrais Hidrelétricas ou PCH, eram operados de forma descentralizada e desordenada, com base apenas no conhecimento dos funcionários envolvidos em tal processo. Desde a crise no setor elétrico brasileiro, ocorrida no ano de 2001, vem aumentando o interesse por empreendimentos relacionados ao conceito de Geração Distribuída e no melhor aproveitamento deste tipo de geração. Isso pode ser obtido de duas formas: investindo na construção de novas instalações e investindo em tecnologia capaz de melhorar o aproveitamento energético das instalações atuais. Para a solução do problema de racionalizar a utilização da estrutura existente, as empresas têm utilizado aplicativos computacionais que auxiliam o operador a tomar decisões com relação à operação do sistema. Como muitos desses aplicativos necessitam informações provenientes de equipamentos distribuídos ao longo do sistema elétrico, torna-se necessário a existência de sistemas de comunicação confiáveis e viáveis economicamente. Diante deste contexto são propostas duas alternativas tecnológicas baseadas em comunicação de dados sobre rede celular para coletar informações elétricas e hídricas de PCHs. Em ambas as propostas são utilizados Controladores Lógicos Programáveis (CLP), os quais são responsáveis por coletar, armazenar e disponibilizar os dados de campo para um sistema SCADA (Supervisory Control and Data Acquisition) localizado no centro de controle (Santos et. al., 2004).

\subsection{Sistema de Aquisição de Dados Via CSD}

Uma das alternativas existentes para se estabelecer um enlace de comunicação CSD entre a estação de supervisão e o CLP é a utilização de telefone celular para recepcionar as conexões no local remoto. Basicamente, o sistema é composto por um servidor responsável por recepcionar as conexões (através de um RAS - Remote Access Services) provenientes do sistema supervisório e efetuar o roteamento dessas requisições ao CLP, bem como pela autenticação e segurança da conexão. O fluxo dos dados se dá a partir de uma requisição de conexão ao servidor remoto, onde será estabelecida a rede IP entre o sistema supervisório e os dispositivos gerenciáveis (neste caso o CLP). Uma vez estabelecida a conexão, o sistema supervisório envia uma requisição de dados ou um conjunto de informações para o endereço IP do dispositivo de 

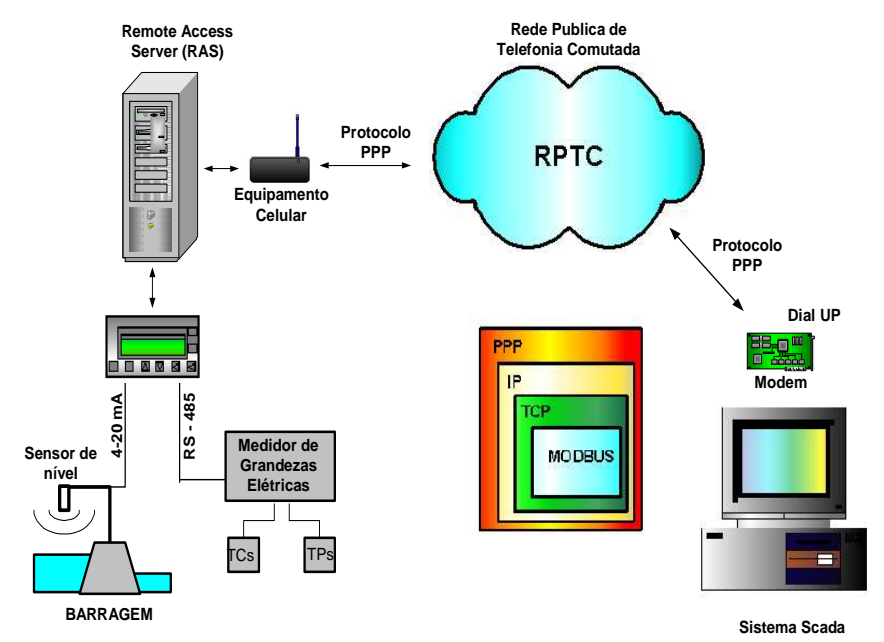

Figura 18: Sistema de aquisição de dados via CSD

destino. Essas requisições são recepcionadas pelo servidor remoto e repassadas para o dispositivo em questão. Esse por sua vez deverá efetuar o processamento requisitado e gerar uma resposta que será encaminhada ao sistema supervisório através do servidor remoto, fechando dessa forma o ciclo de gerenciamento dos dispositivos. A figura 18 apresenta como tal topologia pode ser implementada (Santos et. al., 2004).

\subsection{Sistema de Aquisição de Dados Via GPRS}

Assim como no caso do CSD, o enlace de comunicação de dados utilizando GPRS pode ser implementado de diversas formas. Na solução proposta, estão sendo utilizados dois equipamentos GSM-GPRS. O primeiro é conectado à rede de computadores do centro de operação, onde se encontra o sistema SCADA, e o segundo é implantado junto ao ponto remoto de telemetria, no caso a $\mathrm{PCH}$. Cada equipamento é programado para que sempre exista um enlace de comunicação entre eles, fazendo com que cada requisição gerada pelo SCADA seja interpretada pelo módulo local e repassada para o equipamento remoto. O módulo GSM-GPRS remoto interpreta tal requisição, e repassa-a para o CLP. Após o processamento do CLP o mesmo responde e o caminho inverso é executado. Na figura 19 é apresentado um possível esquema de ligação entre as partes envolvidas no processo de comunicação (Santos et al., 2004).

\section{CONCLUSÕES}

O contínuo avanço das tecnologias de computação móvel em relação à disponibilidade, funcionalidade, facilidade de desenvolvimento de aplicativos e custos de aquisição dos equipamentos, está tornando este tipo de tecnologia cada vez

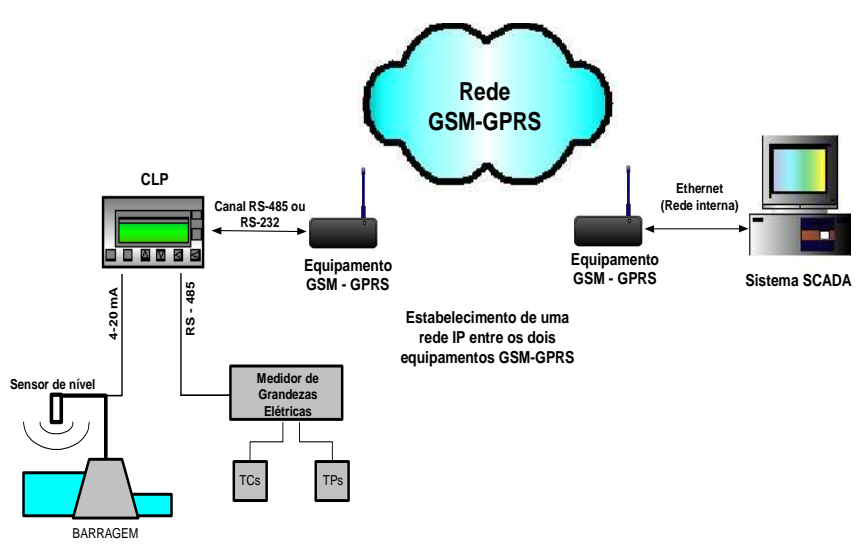

Figura 19: Sistema de coleta de dados de PCH utilizando GPRS.

mais uma opção para a automatização de processos. Desta forma, é natural que funções e processos da indústria de energia elétrica façam uso desse tipo de tecnologia. Dentre as possibilidades de utilização pode-se destacar a coleta e transferência de dados como uma das mais adequadas à utilização da tecnologia da computação móvel. Entretanto, o emprego desse tipo de tecnologia implica, na maioria das vezes, numa mudança significativa do fluxo de informações e análise dos processos, uma vez que atividades rotineiras exercidas por técnicos, as quais são suscetíveis a erros, são substituídas por uma forma automatizada de entrada de dados. Uma das vantagens mais direta do emprego dessa tecnologia está no aumento significativo da qualidade, da confiabilidade e da velocidade das informações manipuladas pelos processos envolvidos. Nesse artigo foram apresentadas três aplicações que demonstram as potencialidades da utilização de tecnologia de computação móvel em sistemas de energia. Duas delas apresentam o uso de PDA para auxílio em rotinas de inspeção e manutenção de sistemas de distribuição, e a terceira apresenta o uso de um sistema de coleta de dados remotos através da rede de telefonia celular para aquisição de informações de uma PCH.

\section{AGRADECIMENTOS}

Este trabalho é resultado de três projetos de Pesquisa e Desenvolvimento (P\&D) ANEEL, suportados pelas empresas RGE Rio Grande Energia S.A., CELESC - Centrais Elétricas de Santa Catarina S.A., HIDROPAN Hidroelétrica Panambi S.A. e ELETROCAR - Centrais Elétricas de Carazinho S.A. Os autores agradecem o suporte técnico e as contribuições dos profissionais dessas empresas que estiveram envolvidos com a execução dos projetos. O primeiro autor agradece a RGE pelo suporte financeiro à realização do seu mestrado. 


\section{REFERÊNCIAS}

CIGRE Task Force 35.07 (2002). The Benefits of Mobile Data How Can They be Realised?. Report of CIGRE Task Force 35.07.

Pretto, C. O.; Rancich, G. V.; Lemos, F. A. B.; Rosa, M. A. (2003a). Forced Outages Information Treatment System and Cause Identification Based on Mobile Computing and Neural Networks. Power Tech, Bolonha, June [em CD].

Pretto, C. O.; Lemos, F. A. B.; Rosa, M. A. (2003b). Computational System For Outage Causes Identification And Analysis For Assessment Of Distribution Systems Reliability Index. Proc. of 17th CIRED - International Conference and Exhibition on Electricity Distribution, Barcelona, Spain. [em CD].

Pretto, C. O.; Lemos, F. A. B.; Rosa, M. A., Faller, R. S. (2005a). New Approach of Electrical Distribution Inspections Routines Using Mobile Technology. Proc. of 18th CIRED - International Conference and Exhibition on Electricity Distribution. Turin, Italy. [em CD]

Pretto, C. O.; Lemos, F. A. B.; Rosa, M. A., Faller, R. S (2005b). Data Acquisition Using Mobile Computing Technology to Enhance Operation and Maintenance Planning. Proc. of 18th CIRED - International Conference and Exhibition on Electricity Distribution, Turin, Italy. [em CD]

Pretto, C. O. (2005c) Sistema de Coleta e Tratamento de Informações sobre Desligamentos Não Programados Baseados em Computadores Móveis. Dissertação de Mestrado. PPGEE - PUCRS, Porto Alegre - RS.

Endrenyi, J.; Aboresheid, S.; Allan, R. N.; Anders, G. J.; Asgarpoor, S.; Billinton, R.; Chowdhury, N.; Dialynas, E. N.; Fipper, M.; Fletcher, R.H.; Grigg, C.; McCalley, J.; Meliopoulos, S.; Mielnik, T. C.; Nitu, P.; Rau, N.; Reppen N. D.; Salvaderi, L.; Schneider, A.; Singh, Ch. (2001). The Present Status of Maintenance Strategies and the Impact of Maintenance on Reliability. IEEE Transactions on Power Systems, Vol. 16, pp. 638 - 646.

Silva Filho, J. M; Ferrari, V.P.; Reichel, A.G.; Nakahata, M.T.; Alemida, A.R (2003) "Desenvolvimento de um Sistema de Coleta de Dados para Inspeção de Usinas, Subestações e Linhas de Transmissão", Anais do II Congresso de Inovação Tecnológica em Energia Elétrica - CITENEL.[em CD]

Rosa, M. A.; Pretto, C. O.; Lemos, F. A. B.; Haffner, S. (2004). Identificação de Causa de Eventos Forçados Utilizando uma Matriz de Pertinência. IEEE Transmission and Distribution, São Paulo [em CD].
Monteiro, M. E.; Moura, E. S.; Drago, A.B.; Santos, L. O. B. S; Amaral, P. F. S.; Filho, J.G.P.; Rosal, P.F.; Bazelatto, G.D. (2003). Sistema para Identificação Local de Equipamentos Instalados nas Linhas de Distribuição por Meio de Coordenadas Geográficas (GPS) e Acesso a Banco de Dados Vai Telefone Celular. Anais do II CITENEL Congresso de Inovação Tecnológica em Energia Elétrica. [em CD]

Meiler, P. (2001) Fundamentals of object-oriented design in $U M L$. Addison Wesley Longman.

Billinton, R.; Oprison, M.; Filippelli, F. and Clark, I. M. (1991). A Reliability Data System for the Reporting of Forced Outages of Distribution Equipment. WESCANEX '91 'IEEE Western Canada Conference on Computer, Power and Communications Systems in a Rural Environment, pp. $267-270$.

Billinton, R. (2002). Economic Cost of Non-Supply. IEEE Power Engineering Society, Winter Meeting, Vol. 2, pp. $959-962$.

Billinton, R. (2002). Assessment of Customers Service Reliability. Proc. of VIII SEPOPE - Symposium of Specialists in Electric Operational and Expansion Planning, Brasília, Brasil.[em CD]

Siemens AG (2003). GPRS Introduction. TM2110E101TM_0005.

Santos, T. T.; Pavão, R. K.; Quadra, A.S.; Lemos, F. A. B. (2004). Aquisição de Dados Remotos Provenientes de Pequenas Centrais Hidroelétricas via Telefonia Celular. IEEE Transmission and Distribution, São Paulo, Brasil [em CD]. 